\title{
Global Solutions for an Extended Class of Hyperbolic Systems of Conservation Laws
}

\author{
J. A. SMOLleR \& J. L. Johnson
}

Communicated by L. CESARI

\section{Introduction}

The purpose of this paper is to extend the results of [7] in order to obtain existence theorems for hyperbolic genuinely non-linear systems of conservation laws of the form

$$
u_{t}+f(u, v)_{x}=0, \quad v_{t}+g(u, v)_{x}=0 .
$$

Here $f$ and $g$ are $C^{2}$ functions of two real variables $u$ and $v$ satisfying ${ }^{\star} f_{v} g_{u}>0$, and $u$ and $v$ are functions of $t$ and $x, t \geqq 0,-\infty<x<\infty$. We shall consider the Cauchy problem for (1.1); i.e., we seek solutions of (1.1) defined in $t \geqq 0$ which also satisfy

$$
(u(x, 0), v(x, 0))=\left(u_{0}(x), v_{0}(x)\right), \quad-\infty<x<\infty,
$$

for some preassigned bounded and measurable functions $u_{0}(x), v_{0}(x)$. It is wellknown, [9], that one cannot, in general, obtain a smooth (i.e., classical) solution to this problem, and we shall therefore seek a "weak" solution. Thus, by a solution to the problem (1.1), (1.2), we mean a pair of bounded, measurable functions $(u(t, x), v(t, x))$ which satisfy

$$
\begin{aligned}
& \iint_{t \geq 0}\left[u \phi_{t}+f(u, v) \phi_{x}\right] d x d t+\int_{t=0} u_{0} \phi d x=0 \\
& \iint_{t \geq 0}\left[v \phi_{t}+g(u, v) \phi_{x}\right] d x d t+\int_{t=0} v_{0} \phi d x=0
\end{aligned}
$$

for all smooth functions $\phi=\phi(t, x)$ having compact support.

In order to obtain an existence theorem for the problem (1.1), (1.2), we shall assume that the initial data satisfies a certain order condition which was introduced in [11] and which was also used in [7] to obtain existence theorems. Briefly, this condition states that certain initial data gives rise to a specific form of Riemann problem. Thus, if we let $\left(u_{i}, v_{i}\right)=\left(u_{0}\left(x_{i}\right), v_{0}\left(x_{i}\right)\right), i=1,2$, for $x_{1}<x_{2}$, then we assume that Riemann's problem for $(1.1)$ with initial data $\left(u_{0}(x), v_{0}(x)\right)=\left(u_{1}, v_{1}\right)$ if $x<0$, and $\left(u_{0}(x), v_{0}(x)\right)=\left(u_{2}, v_{2}\right)$ if $x>0$, is solvable by a shock wave of one

* This condition implies that the system (1.1) is hyperbolic.

13 Arch. Rational Mech. Anal., Vol. 32 
characteristic family and a rarefaction wave of the opposite family ${ }^{\star}$. In addition to these assumptions on the initial data, we shall also assume that the system satisfies two other conditions which we shall now describe. First we shall assume a hypothesis which implies that the system (1.1) locally satisfies the GLIMM-LAX shock interaction condition. This condition was introduced in [5], is characterized in the Appendix, and states that the interaction of two shock waves of the same characteristic family produce a shock wave of this family plus a rarefaction wave of the opposite characteristic family. In addition to this condition, we shall also assume a condition, condition $(L)$, which assures us that all shock waves under consideration arise from the intersection of two characteristics from exactly one characteristic family. This requirement is always true locally and we shall give several natural conditions which assure us that this condition holds globally. We remark that these two assumptions hold for the systems considered in [7] and [11]. In fact, they are precisely the conditions which enable us to extend the methods of [7] to the more general systems (1.1).

The plan of this paper is first to construct through each point $P_{0}$, in some open set in $u-v$ space where our assumptions hold, two global curves, called the wave and shock curves, which consist of states which can be connected in $P_{0}$ by a rarefaction wave of one characteristic family and a shock wave of the other characteristic family, respectively. We then show that our assumptions assure us that these curves have properties analogous to those considered in [7]. As a consequence of these properties we derive a necessary contition that any uniqueness conditions must satisfy. Using these properties of our curves we can apply the methods of [7] to obtain a solution to the problem (1.1), (1.2). The solution is a limit of a sequence of solutions of (1.1) with step data. These approximating solutions are uniformly bounded and have uniformly bounded variation, locally, in the sense of TONELli-Cesari, [2], with respect to two independent, not necessarily orthogonal, directions. From this it follows that the sequence of approximating solutions is compact in the topology of $L_{1}$-convergence on compacta, and hence contains a subsequence converging to a solution of the original problem (1.1), (1.2). We shall then show that we can also obtain a solution to (1.1), (1.2), under the same hypotheses, using the difference scheme introduced by J. GLIMM in [4]. This shows that the GLIMM scheme can be used to solve certain Cauchy problems where the variation of the initial data is arbitrarily large. We end the paper with some concluding remarks and examples.

\section{The Geometry of the Wave and Shock Curves}

In this section we shall construct the wave and shock curves and then derive their main properties. This analysis is basic in our work, and we hope it will also prove useful in obtaining existence and uniqueness theorems for more general initial data.

Let $F$ be the mapping from $E^{2}$ into $E^{2}$ defined by $F:(u, v) \rightarrow(f(u, v), g(u, v))$, and denote by $\operatorname{dF}(u, v)$ the Fréchet derivative (Jacobian) of $F$. We assume that

$$
f_{v} g_{u}>0
$$

\footnotetext{
* The directions of these families are fixed, independent of the $x_{i}$ 's.
} 
in an open set $U \subseteq E^{2}$, and for definiteness we shall assume that

$$
f_{v}<0 \text { and } g_{u}<0 \text { in } U \text {. }
$$

Then $d F(u, v)$ has real and distinct eigenvalues $\lambda_{2}(u, v)>\lambda_{1}(u, v)$ for all $(u, v) \in U$. We denote by $r_{i}(u, v), i=1,2$, the corresponding right eigenvectors which we choose to write in the form *

$$
r_{1}=\left(1, a_{1}\right)^{t}, \quad r_{2}=\left(-1,-a_{2}\right)^{t}, \quad \text { where } \quad a_{i}=g_{u} /\left(\lambda_{i}-g_{v}\right), \quad i=1,2 .
$$

Following LAX [9], we assume that the system (1.1) is genuinely non-linear in $U$. This means that $d \lambda_{i}\left(r_{i}\right) \neq 0$ in $U, i=1,2$, and we normalize the $r_{i}$ by

$$
d \lambda_{i}(u) r_{i}(u)>0, \quad u \in U, \quad i=1,2 .
$$

Let

$$
l_{1}=\left(-a_{2}, 1\right), \quad l_{2}=\left(-a_{1}, 1\right),
$$

be left eigenvectors of $d F(u, v)$, normalized by $l_{i} r_{i}>0, i=1,2$. We shall also assume that the system satisfies

$$
l_{j}(u, v) d^{2} F\left(r_{i}(u, v), r_{i}(u, v)\right)>0, \quad(u, v) \in U, \quad i, j=1,2, \quad i \neq j,
$$

where $d^{2} F$ is the second Fréchet derivate of $F$. (See [2] for a discussion of derivatives of vector-valued mappings.) It is shown in the Appendix that for sufficiently weak shocks this implies the GLIMM-LAX shock interaction condition [5], which states that the interaction of two shocks of the same characteristic family produces a shock of that characteristic family plus a rarefaction wave of the other characteristic family. With these normalizations for $l_{i}$ and $r_{i}$, it is also shown in the Appendix that the condition (2.4) for genuine non-linearity is equivalent to

$$
l_{i}(u, v) d^{2} F\left(r_{i}(u, v), r_{i}(u, v)\right)>0, \quad(u, v) \in U, \quad i=1,2 .
$$

Thus we can write (2.4) and (2.6) in the form

$$
l_{j}(u, v) d^{2} F\left(r_{i}(u, v), r_{i}(u, v)\right)>0, \quad(u, v) \in U, \quad i, j=1,2 .
$$

We are now in a position to construct the wave and shock curves. Thus, let $P_{0}=\left(u_{0}, v_{0}\right)$ be a point in $U$. We shall construct two curves $v=w\left(u ; P_{0}\right)$ and $v=s\left(u ; P_{0}\right)$ which represent states which can be connected to $P_{0}$ by rarefaction waves and shock waves. We shall first consider the case of rarefaction waves. It is well-known [10] that there exists a transformation of independent variables $(u, v) \rightarrow(r, s)$ which puts the system (1.1) into Riemann-invariant form:

$$
\begin{aligned}
& r_{t}+\lambda_{2} r_{x}=0 \\
& s_{t}+\lambda_{1} s_{x}=0 .
\end{aligned}
$$

A rarefaction wave is an open region in the $x-t$ plane in which one of the Riemann-invariants $r$ or $s$ is constant. From (2.8) we see that $r$ and $s$ are constant along curves $\dot{x}=\lambda_{2}$ and $\dot{x}=\lambda_{1}$, respectively. We shall refer to these curves as

* We make this particular choice in order that our diagrams in $u-v$ space should have a familiar form (cf. [7]). 
characteristics of the second and first characteristic family, respectively. A 1 (respectively 2)-rarefaction wave, or a rarefaction wave of the first (respectively second) characteristic family is an open region in the $x-t$ plane in which $r$ (respectively $s$ ) is constant. Since we shall only be concerned with rarefaction waves from a single family, we shall just consider 1 -rarefaction waves. In deriving (2.8) from (1.1), it was shown [10] that $l_{2}$ is parallel to $d r(u, v)=\left(r_{u}, r_{v}\right)$, so that $r_{1}$ is perpendicular to $d r(u, v)$. Hence the curve $r(u, v)=$ constant satisfies the differential equation

$$
\frac{d v}{d u}=a_{1} .
$$

Let the solution of (2.9) through $P_{0}$ be denoted by $v=w\left(u ; P_{0}\right)=w(u)$. Since $f_{v} g_{u}>0$ and $g_{u}<0$, we see that along $v=w(u), v$ is an increasing function of $u$. Since from $(2.4) d \lambda_{1}(u, v)\left(r_{1}(u, v)\right)>0$, we see that $\lambda_{1}$ increases along this curve and hence each point on this curve satisfies the LAX condition for rarefaction waves (equation (8.7) of [9]). We shall next show that along this curve $d^{2} v / d u^{2}<0$. To see this, note that $d^{2} v / d u^{2}=\left(a_{1}\right)_{u}+a_{1}\left(a_{1}\right)_{v}$. If we write $a_{1}=g_{u} /\left(\lambda_{1}-g_{v}\right)$, we get

$$
\begin{aligned}
\left(\lambda_{1}-g_{v}\right) \frac{d^{2} v}{d u^{2}} & =g_{u u}+2 a_{1} g_{u v}+a_{1}^{2} g_{v v}-a_{1}\left[\left(\lambda_{1}\right)_{u}+a_{1}\left(\lambda_{1}\right)_{v}\right] \\
& =d^{2} g\left(r_{1}, r_{1}\right)-a_{1}\left[\left(\lambda_{1}\right)_{u}+a_{1}\left(\lambda_{1}\right)_{v}\right] .
\end{aligned}
$$

If we now write $a_{1}=\left(\lambda_{1}-g_{u}\right) / f_{v}$, we similarly get

$$
f_{v} \frac{d^{2} v}{d u^{2}}=-d^{2} f\left(r_{1}, r_{1}\right)+\left(\lambda_{1}\right)_{u}+a_{1}\left(\lambda_{1}\right)_{v} .
$$

It follows that

$$
\left(\lambda_{1}-g_{v}+a_{1} f_{v}\right) \frac{d^{2} v}{d u^{2}}=l_{2} d^{2} F\left(r_{1}, r_{1}\right),
$$

and since $\lambda_{1}-g_{v}+a_{1} f_{v}=\lambda_{1}-\lambda_{2}<0$ we see from $(2.5)$ that $d^{2} v / d u^{2}<0$. As a consequence of this convexity, we see that $v=w(u)$ is defined for all $u \geqq u_{0}$ for which $(u, w(u)) \in U$. We have thus proved the following theorem.

Theorem 2.1. Let the system (1.1) satisfy (2.2) and (2.7) in an open subset $U \subseteq E^{2}$. Then through any point $P_{0} \in U$, there exists an increasing (respectively decreasing), convex downward (respectively upward) curve $v=w\left(u ; P_{0}\right)$ defined for all $u \geqq u_{0}$ (respectively $\left.u \leqq u_{0}\right)$ for which $\left(u, w\left(u ; P_{0}\right)\right) \in U$, having the property that any point on this curve is a state which can be connected to $P_{0}$ on the right by a 1 (respectively 2)-rarefaction wave.

We shall next consider the shock curves. These curves satisfy the RankineHugoniot conditions

$$
\sigma\left(u-u_{0}\right)=f(u, v)-f\left(u_{0}, v_{0}\right), \quad \sigma\left(v-v_{0}\right)=g(u, v)-g\left(u_{0}, v_{0}\right)
$$

where $\sigma=\sigma\left(u, v ; u_{0}, v_{0}\right)$ is the shock speed. We eliminate $\sigma$ in these equations to get

$$
\left(u-u_{0}\right)\left[g(u, v)-g\left(u_{0}, v_{0}\right)\right]=\left(v-v_{0}\right)\left[f(u, v)-f\left(u_{0}, v_{0}\right)\right] .
$$

We seek $a$ and $b$ so that we can write

$$
v=v_{0}+a\left(u-u_{0}\right)+\frac{1}{2} b\left(u-u_{0}\right)^{2}+O\left(\left(u-u_{0}\right)^{3}\right), \quad u \geqq u_{0} .
$$


To do this, we make the change of variable $v-v_{0}=\left(u-u_{0}\right) \phi(u)$ where $\phi(u)=$ $a+b\left(u-u_{0}\right)+y(u)$, and substitute in (2.10) to get $\left(u-u_{0}\right)^{2} H(u, y)=0$, where

$$
\begin{aligned}
H(u, y)= & g_{u}+\phi\left(g_{v}-f_{u}\right)-\phi^{2} f_{v} \\
& +\left(u-u_{0}\right)\left[\frac{1}{2}\left(g_{u u}+\phi^{2} g_{u v}-\phi f_{u u}-\phi^{3} f_{v v}\right)+\phi g_{u v}-\phi^{2} f_{u v}\right]+O\left(\left(u-u_{0}\right)^{2}\right)
\end{aligned}
$$

and the derivatives of $f$ and $g$ are evaluated at $\left(u_{0}, v_{0}\right)$. Then

$$
\begin{aligned}
& H\left(u_{0}, 0\right)=g_{u}+a\left(g_{v}-f_{u}\right)-a^{2} f_{v}, \\
& H_{y}\left(u_{0}, 0\right)=g_{v}-f_{u}-2 a f v .
\end{aligned}
$$

We choose $a$ such that $H\left(u_{0}, 0\right)=0$. Since we are interested in shocks of the second characteristic family, we have $a=a_{2} .^{\star}$ For this choice of $a$, the hyperbolicity assumption implies that $H_{y}\left(u_{0}, 0\right) \neq 0$. The implicit function theorem then implies that we can find a function $y(u)$ satisfying our requirements. A similar computation for $b$ shows that $\left(\lambda_{2}-\lambda_{1}\right) b=l_{1} d^{2} F\left(r_{2}, r_{2}\right)$. We have thus shown that the shock curve (2.10) for 2-shocks can be written in the form (2.11) where

$$
a=g_{u} /\left(\lambda-g_{v}\right)
$$

and where we are dropping the subscripts and writing $\lambda=\lambda_{2}, a=a_{2}$, and

$$
b=\frac{l_{1} d^{2} F\left(r_{2}, r_{2}\right)}{\lambda_{2}-\lambda_{1}}
$$

so that $a<0$ and $b>0$. From (2.10) and (2.11) we see that the differential equation of the shock curve is

$$
\frac{d v}{d u}= \begin{cases}\frac{g(u, v)-g\left(u_{0}, v_{0}\right)+\left(u-u_{0}\right) g_{u}-\left(v-v_{0}\right) f_{u}}{f(u, v)-f\left(u_{0}, v_{0}\right)+\left(v-v_{0}\right) f_{v}-\left(u-u_{0}\right) g_{v}}, & u \neq u_{0} \\ a, & u=u_{0} .\end{cases}
$$

We let $\left(u_{1}, v_{1}\right)$ be a point on the shock curve close to $\left(u_{0}, v_{0}\right)$ and we solve the problem

$$
\frac{d v}{d u}=\frac{g(u, v)-g\left(u_{0}, v_{0}\right)+\left(u-u_{0}\right) g_{u}-\left(v-v_{0}\right) f_{u}}{f(u, v)-f\left(u_{0}, v_{0}\right)+\left(v-v_{0}\right) f_{v}-\left(u-u_{0}\right) g_{v}}, \quad v\left(u_{1}\right)=v_{1} .
$$

Denote the solution of this problem by $v=s\left(u ; P_{0}\right)=s(u)$. We shall now need some additional notation. Let $v=s\left(u ; P_{0}\right)$; then we define

$$
\begin{aligned}
\sigma=\sigma(u, v)=\sigma\left(u, v ; P_{0}\right) & =\frac{f(u, v)-f\left(u_{0}, v_{0}\right)}{u-u_{0}}=\frac{g(u, v)-g\left(u_{0}, v_{0}\right)}{v-v_{0}} \\
h=h(u, v)=h\left(u, v ; P_{0}\right) & =\frac{g(u, v)-g\left(u_{0}, v_{0}\right)+\left(u-u_{0}\right) g_{u}-\left(v-v_{0}\right) f_{v}}{f(u, v)-f\left(u_{0}, v_{0}\right)+\left(v-v_{0}\right) f_{v}-\left(u-u_{0}\right) g_{v}} \\
& =\frac{\left(u-u_{0}\right) g_{u}+\left(\sigma-f_{u}\right)\left(v-v_{0}\right)}{\left(v-v_{0}\right) f_{v}+\left(\sigma-g_{v}\right)\left(u-u_{0}\right)}
\end{aligned}
$$

$\frac{d}{d \mu}=\frac{\partial}{\partial u}+h \frac{\partial}{\partial v}=$ differentiation along $v=s\left(u ; P_{0}\right)$

$\star$ In [9] it is shown that the 2-shock curve and the 2-rarefaction wave curve together form a $C^{2}$ function at $P_{0}$. 
We define an $i$-shock, or a shock of $i^{\text {th }}$ characteristic family, $i=1,2$, to be a discontinuity $x=x(t)$ of the function $(u(t, x), v(t, x))$ satisfying the RankineHugoniot condition and the single equality

$$
\lambda_{i}(u(x+0, t), v(x+0, t))<\dot{x}(t)<\lambda_{i}(u(x-0, t), v(x-0, t))
$$

where $\dot{x}(t)=\sigma(u(x+0, t), v(x+0, t) ; u(x-0, t), v(x-0, t))$ is the shock speed. This definition differs slightly from that of LAX [9] in that we do not now require the additional condition $\lambda_{1}(u(x-0, t), v(x-0, t))<\dot{x}(t)$ to hold for 2-shocks and the additional condition $\lambda_{2}(u(x+0, t), v(x+0, t))>\dot{x}(t)$ to hold for 1 -shocks. We shall have more to say about these conditions later. With this definition we can prove the following theorem.

Theorem 2.2. Let the system (1.1) satisfy (2.2) and (2.7) in $U \subseteq E^{2}$. Then through each point $P_{0}=\left(u_{0}, v_{0}\right) \in U$ there exists a decreasing (respectively increasing) curve $v=s(u)=s\left(u ; P_{0}\right)$ having the property that any point on this curve is a state which can be connected on the right to $P_{0}$ by a shock wave of the second (respectively first) characteristic family.

Proof. We shall only give the proof for 2-shocks, and we therefore have to show

$$
\lambda\left(u_{0}, v_{0}\right)>\sigma(u, v)>\lambda(u, v) .
$$

From [9] we have $\sigma(u, v)>\lambda(u, v)$ for $(u, v)$ close to $\left(u_{0}, v_{0}\right), u>u_{0}$. If this inequality is not true all along $v=s(u)$, let $P_{1}=\left(u_{1}, v_{1}\right)$ be the first point of equality, i. e., $\sigma\left(P_{1}\right)=\lambda\left(P_{1}\right), u_{1}>u_{0}$. Then

$$
h\left(P_{1}\right)=\frac{\left(u_{1}-u_{0}\right) a\left(\lambda-g_{v}\right)+a f_{v}\left(v_{1}-v_{0}\right)}{\left(v_{1}-v_{0}\right) f_{v}+\left(\lambda-g_{v}\right)\left(u_{1}-u_{0}\right)}=a\left(P_{1}\right)
$$

since it is easy to see that the denominator is non-zero at $P_{1}$ * ${ }^{*}$ Furthermore, $d \sigma / d \mu=0$ at $P_{1}$ so that

$$
\left.\frac{d}{d \mu}(\sigma-\lambda)\right|_{P_{1}}=-\left.\frac{d \lambda}{d \mu}\right|_{P_{1}}=-\left.\left(\lambda_{u}+a \lambda_{v}\right)\right|_{P_{1}}>0
$$

in view of (2.4). This contradicts the definition of $P_{1}$. Hence $\sigma(u, v)>\lambda(u, v)$ along $v=s(u)$. We note that this implies that $u>u_{0}$ and $v<v_{0}$ along $v=s(u)$ since we known that these are true locally near $\left(u_{0}, v_{0}\right)$ and $\sigma(u, v)>\lambda(u, v)$ implies that $u=s(v)$ cannot have any vertical or horizontal tangents.

We shall now prove that $\sigma$ decreases along $v=s(u)$, and this will imply that $\lambda\left(P_{0}\right)>\sigma(u, v)$ since $\sigma\left(P_{0}\right)=\lambda\left(P_{0}\right)$, (see [9]).

To to this, we first note that from [9], $\sigma$ decreases along $v=s(u)$ close to $P_{0}$. If $d \sigma / d \mu$ were ever zero at a point, then at this point $f_{u}-\sigma+h f_{v}=0$. If we write this out using the definition of $h$, it would imply that $\sigma=\lambda$, contrary to what we have just proved. Hence $d \sigma / d \mu<0$ and the proof of the theorem is complete.

* In fact, if $\left(v_{1}-v_{0}\right) f_{v}+\left(\lambda-g_{v}\right)\left(u_{1}-u_{0}\right)=0$, then it follows from the definition of $P_{1}$, that $P_{1}$ must be the first point where this holds. Hence $h\left(P_{1}\right)<0$ so that $u_{1}-u_{0}>0$ and $v_{1}-v_{0}>0$. But $f_{v}<0$ and $\lambda-g_{v}>0$, and this is impossible. 
The next theorem continues our study of the properties of the shock curves.

Theorem 2.3. The shock curve $v=s\left(u ; P_{0}\right)$ satisfies

$$
\begin{gathered}
a(u, v)>h\left(u, v ; u_{0}, v_{0}\right)>\frac{v-v_{0}}{u-u_{0}} \\
\frac{d}{d \mu}\left(\frac{v-v_{0}}{u-u_{0}}\right)>0 \\
\frac{v_{2}-v}{u_{2}-u}>\frac{v_{1}-v}{u_{1}-u} \quad \text { if } u>u_{2}>u_{1} .
\end{gathered}
$$

It is interesting to see what these conditions mean geometrically. For example, the first equality in (2.17) says that a shock curve originating at a point on the original shock curve has the slope of its tangent vector greater than the slope of the original shock curve at the point. Thus the new shock curve starts out "breaking to the right", and this implies that the GLIMM-LAX shock interaction condition holds locally all along the shock curve (and not just in a neighborhood of $P_{0}$ ). Similarly, (2.19) implies that the shock curve is convex upward.

Proof. We shall first compute $d a / d \mu$ at $P_{0}$. To do this, we use (2.12) to get

$$
\left(\lambda-g_{v}\right) \frac{d a}{d \mu}=d^{2} g\left(r_{2}, r_{2}\right)-a\left(\lambda_{u}+a \lambda_{v}\right) .
$$

If we write $a=\left(\lambda-f_{u}\right) / f_{v}$, then we get

Hence

$$
f_{v} \frac{d a}{d \mu}=-d^{2} f\left(r_{2}, r_{2}\right)+\left(\lambda_{u}+a \lambda_{v}\right) .
$$

Also, from (2.11)

$$
\left.\frac{d a}{d \mu}\right|_{P_{0}}=\frac{l_{1} d^{2} F\left(r_{2}, r_{2}\right)}{\lambda_{2}-\lambda_{1}}=b
$$

so that

$$
\left.\frac{d}{d \mu}\left(\frac{v-v_{0}}{u-u_{0}}\right)\right|_{P_{0}}=\frac{b}{2}
$$

$$
\frac{d}{d \mu}\left[\left(\frac{v-v_{0}}{u-u_{0}}-a\right)\right]_{P_{0}}=-\frac{b}{2}<0,
$$

and this implies that $a>\left(v-v_{0}\right) /\left(u-u_{0}\right)$ for $u$ close to $u_{0}$ since $\left(v-v_{0}\right) /\left(u-u_{0}\right) \rightarrow$ $a\left(P_{0}\right)$ as $u \rightarrow u_{0}$, again by (2.9). The rest of the proof of this theorem proceeds in a manner analogous to theorem (2.3) of [7]. We leave the details to the reader.

Corollary 2.4. The shock curve $v=s\left(u ; P_{0}\right)$ is defined for all $u \geqq u_{0}$, as long as $\left(u, s\left(u ; P_{0}\right)\right) \in U$.

Proof. Inequality (2.19) of the last theorem shows that the shock curve is convex upward. Since the shock curve is also decreasing, it cannot escape; i.e., there is no finite $u_{1}>u_{0}$ for which $\lim s\left(u ; P_{0}\right)=-\infty$ as $u \rightarrow u_{1}$. 
We shall next obtain an ordering principle which is the basic step in our existence theorem. Let $P_{0}=\left(u_{0}, v_{0}\right) \in U$ and let

$$
C\left(P_{0}\right)=\left\{(u, v) \in U: s\left(u ; P_{0}\right) \leqq v \leqq w\left(u ; P_{0}\right), u \geqq u_{0}\right\} .
$$

We wish to prove the analogue of theorem (2.4) in [7] which states that if $P_{1} \in C\left(P_{0}\right)$ then $C\left(P_{1}\right) \subseteq C\left(P_{0}\right)$. However, we cannot prove this theorem without making an additional assumption, which, along with our previous assumptions, is necessary and sufficient for this theorem to be true. This additional assumption is

(L) For each $P_{0} \in U$, if $v=s\left(u ; P_{0}\right),(u, v) \in U$, then $\sigma\left(u, v ; P_{0}\right)>\lambda_{1}\left(P_{0}\right)$.

This condition assures us that all 2-shocks under consideration, arise from the intersection of characteristics from the second characteristic family, and not from the intersection of characteristics of the first characteristic family. Condition $(L)$ is part of the definition of shocks in [9]. We shall now give some sufficient conditions in order that $(L)$ holds.

Lemma 2.5. (1) Condition ( $L$ ) holds for sufficiently weak shocks in $U$.

(2) Condition $(L)$ holds if any of the following conditions hold in $U$ :

(a) $\lambda_{2} \geqq 0 \geqq \lambda_{1}$

(b) $\left(\lambda_{1}\right)_{v} \leqq 0$

(c) $f_{u u} \geqq 0$ and $f_{u v} \leqq 0$ or $g_{v u} \geqq 0$ and $g_{v v} \leqq 0$.

Proof. In [9] it is shown that $\sigma\left(P_{0}\right)=\lambda_{2}\left(P_{0}\right)$ so that $\sigma\left(P_{0}\right)>\lambda_{1}\left(P_{0}\right)$ whence $(L)$ holds for $(u, v)$ close to $P_{0}$. If (a) holds, then from $(2.16), \sigma(u, v)>\lambda_{2}(u, v) \geqq$ $0 \geqq \lambda_{1}\left(P_{0}\right)$. If (b) holds, then from (2.17) and (2.4)

$$
\frac{d \lambda_{1}}{d \mu}=\left(\lambda_{1}\right)_{u}+h\left(\lambda_{1}\right)_{v} \geqq\left(\lambda_{1}\right)_{u}+a_{1}\left(\lambda_{1}\right)_{v}>0
$$

so that $\lambda_{1}$ increases along the shock curve. Hence by (2.16)

$$
\sigma(u, v)>\lambda_{2}(u, v)>\lambda_{1}(u, v)>\lambda_{1}\left(P_{0}\right) .
$$

Finally, if $f_{u v} \leqq 0$ and $f_{u u} \geqq 0$, then

$$
\frac{d}{d \mu} f_{u}=f_{u u}+h f_{u v} \geqq 0 .
$$

Hence $f_{u}$ is non-decreasing along the shock curve so that

$$
\sigma(u, v)>f_{u}(u, v) \geqq f_{u}\left(u_{0}, v_{0}\right)>\lambda_{1}\left(u_{0}, v_{0}\right) .
$$

Similarly, $(L)$ holds if $g_{v u} \geqq 0$ and $g_{v v} \leqq 0$. This completes the proof.

We note that conditions (a) and (c) both hold for the systems considered in [7] and [11].

Theorem 2.6 (Ordering Principle). Suppose that assumptions (2.2) and (2.7) and condition $(L)$ hold in $U$. Let $P_{0}=\left(u_{0}, v_{0}\right) \in U$ and let $P_{1}=\left(u_{1}, v_{1}\right) \in C\left(P_{0}\right)$. Then $C\left(P_{1}\right) \subseteq C\left(P_{0}\right)$. 
Proof. It suffices to show that the shock curve $v=s\left(u ; P_{1}\right)$ never goes below the shock curve $v=s\left(u ; P_{0}\right)$. The case where $P_{1}$ lies on $v=s\left(u ; P_{0}\right)$ was proved in $[8]^{\star}$. For the general case, we need two lemmas.

Lemma 2.7. Let $P_{2}=\left(u_{2}, v_{2}\right) \in C\left(P_{0}\right)$. Then there exists a point $P_{3}$ on the wave curve through $P_{0}$ such that $P_{2}$ lies on the shock curve originating at $P_{3}$.

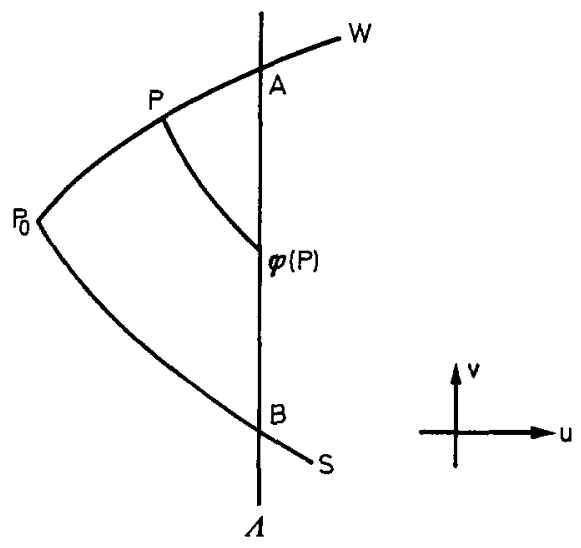

Fig. 1

Proof. In our notation, we are to show that there exists a point $u_{3} \geqq u_{0}$ for which $v_{2}=s\left(u_{2} ; u_{3}, w\left(u_{3} ; P_{0}\right)\right)$. Let $\Lambda$ be the vertical line $u=u_{2}$, let $S$ be the shock curve $v=s\left(u ; P_{0}\right)$ and let $W$ be the wave curve $v=w\left(u ; P_{0}\right)$. Consider the mapping $\phi$ from $W$ to $A$ defined by

$$
\phi:\left(u ; w\left(u ; P_{0}\right)\right) \rightarrow\left(u_{2} ; s\left(u_{2} ; u, w\left(u ; P_{0}\right)\right)\right),
$$

that is, $\phi$ maps a point $P$ on $W$ into the point of intersection of $\Lambda$ with the shock curve originating at $P$ (see Figure 1). Since $-\infty<h<0$ along the shock curves, we see that the shock curves originating at a point on $W$ with $u_{0} \leqq u<u_{2}$ cut $A$ transversally. This implies that $\phi$ is continuous. Let $A$ and $B$ be the points of intersection of $A$ with $W$ and $S$ respectively. Since the region enclosed by $P_{0} A B$ is compact, the slopes of the shock curves originating on $W$, in this region, are bounded. Hence points on $W$ close to $A$ must map under $\phi$ into points above $P_{2}$ on $A$. Also since $\phi\left(P_{0}\right)=B$ and $W$ is connected, there must be a point $P_{3}$ on $W$ for which $\phi\left(P_{3}\right)=P_{2}$. This completes the proof of the lemma.

The next lemma applies this result to characterize when the ordering principle holds.

Lemma 2.8. $C\left(P_{1}\right) \subseteq C\left(P_{0}\right)$ for each $P_{1} \in C\left(P_{0}\right)$ if and only if for each $P_{2}$ on the wave curve $v=w\left(u ; P_{0}\right)$, the shock curve $v=s\left(u ; P_{2}\right)$ does not go through the shock curve $v=s\left(u ; P_{0}\right)$.

* We note that this case was proved independently of $(L)$ and shows that the interaction of two 2-shocks produces a 2-shock and a 1-rarefaction wave. In other words, the GLImM-LAX shock interaction condition is valid globally in $U$. 
Proof. The condition is certainly necessary. We must thus show that the condition is sufficient. Let $W$ and $S$ be the wave and shock curves $v=w\left(u ; P_{0}\right)$ and $v=s\left(u ; P_{0}\right)$, respectively. Suppose that there were a point $\left(u^{\prime}, v^{\prime}\right)=P^{\prime} \in C\left(P_{0}\right)$ with $v^{\prime}>s\left(u^{\prime} ; P_{0}\right)$ for which the shock curve $v=s\left(u ; P^{\prime}\right)$ intersected $S$. By Lemma 2.7 , there is a point $P_{2}$ on $W$ such that the shock curve $v=s\left(u ; P_{2}\right)$ passes through $P^{\prime}$. In view of Theorem 2.3 , the shock curve $v=s\left(u ; P_{2}\right)$ meets $S$, and by previous results this curve actually passes through $S$ (see Figure 2). This is a contradiction and the proof of the lemma is complete.

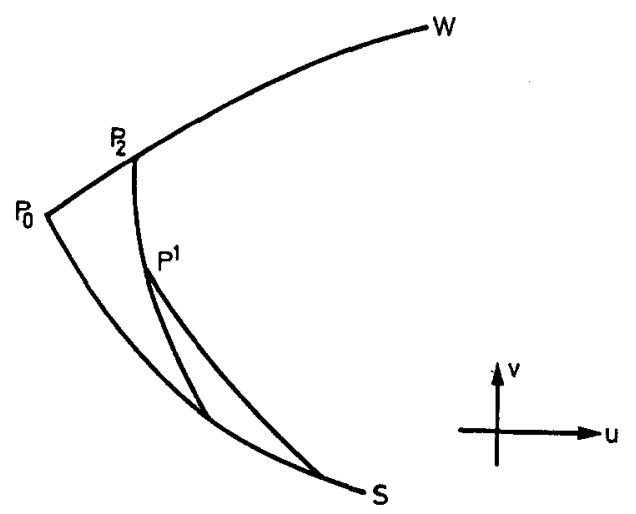

Fig. 2

We note that condition $(L)$ was not used in the proof of these lemmas.

We shall now complete the proof of Theorem 2.6. The idea of the proof is as follows. Let $P_{0} \in U$, let $W$ and $S$ be as before the wave and shock curves $v=w\left(u ; P_{0}\right)$ and $v=s\left(u ; P_{0}\right)$, respectively, and let $P \in S$. We want to show that as $P_{0}$ moves along $W$ to $P_{0}^{\prime}$ in the direction of increasing $u$, then the point of intersection of the shock curve $v=s\left(u ; P_{0}^{\prime}\right)$ with the wave curve $v=w(u ; P)$ also moves in the direction of increasing $u$. It will then follow that the shock curve $v=s\left(u ; P_{0}^{\prime}\right)$ cannot meet the shock curve $v=s\left(u ; P_{0}\right)$, and so we will be done by Lemma 2.8 .

Thus, let $G\left(P, P_{0}, \sigma\right)$ be the mapping from $U \times U \times E^{\prime}$ into $E^{2}$ defined by

$$
G\left(P, P_{0}, \sigma\right)=F(P)-F\left(P_{0}\right)-\sigma\left(P-P_{0}\right) .
$$

Then $P$ and $P_{0}$ can be joined by a 2-shock if there is a $\sigma$ satisfying (2.16) for which $G\left(P, P_{0}, \sigma\right)=0$. We claim that in view of our previous remarks it suffices to show that there exist $\alpha>0$ and $t$ real such that

$$
d G\left(P, P_{0}, \sigma\right)\left[\alpha r_{1}(P), r_{1}\left(P_{0}\right), t\right]=0 .
$$

To see this, we represent the points of $W$ by $\phi_{\tau} P_{0}, \tau \geqq 0$. If we let $\phi_{\theta} P, \theta \geqq 0$, represent the points on the wave curve $v=w(u ; P)$, then for each $\tau$ there exist $\theta=\theta(\tau)$ and $\sigma=\sigma(\tau)$ such that $\star$

$$
G(\tau) \equiv G\left(\phi_{\theta(\tau)} P, \phi_{\tau} P_{0}, \sigma(\tau)\right)=0 .
$$

\footnotetext{
* The shock curves negative slopes and the wave curves have positive slopes.
} 
We are to show that $\theta^{\prime}(\tau)>0$. Thus, if we differentiate this with respect to $\tau$, we get

$$
d G(\tau)\left[\theta^{\prime}(\tau) r_{1}(P), r_{1}\left(P_{0}\right), \sigma^{\prime}(\tau)\right]=0
$$

which is equivalent to (2.20). Now expanding (2.20) gives

$$
\alpha\left(\lambda_{1}(P)-\sigma\right) r_{1}(P)-\left(\lambda_{1}\left(P_{0}\right)-\sigma\right) r_{1}\left(P_{0}\right)-t\left(P-P_{0}\right)=0 .
$$

We must show that $\alpha\left(=\alpha\left(P, P_{0}\right)\right)$ is always positive. Our hypothesis $(L)$ implies that $\lambda_{1}\left(P_{0}\right)-\sigma<0$ so that if $\alpha=0$ we would have $P-P_{0}$ parallel to $r_{1}\left(P_{0}\right)$ which is impossible since

$$
\text { slope }\left(P-P_{0}\right)<a_{2}(P)<0<a_{1}\left(P_{0}\right)=\text { slope } r_{1}\left(P_{0}\right)
$$

from (2.3) and Theorem 2.3. Next, from hyperbolicity and (2.16) we obtain $\lambda_{1}(P)-\sigma<0$, and this along with $(L)$ shows that $\alpha>0$ for $P$ close to $P_{0}$. Finally, if we write (2.21) as

$$
\alpha r_{1}(P)=\frac{\lambda_{1}\left(P_{0}\right)-\sigma}{\lambda_{1}(P)-\sigma} r_{1}\left(P_{0}\right)+\frac{t}{\lambda_{1}(P)-\sigma}\left(P-P_{0}\right),
$$

we see that $\alpha r_{1}(P)$ is continuous, and since $r_{1}(P)$ is continuous, it follows that $\alpha$ is continuous. Thus $\alpha>0$ for all $P, P_{0}$ and this completes the proof of the theorem.

We remark that an alternate, less geometrical, way to obtain this last part is to show that

$$
\frac{\partial s}{\partial u_{0}}\left(u ; u_{0}, w\left(u_{0} ; \alpha, \beta\right)\right)=n\left[\lambda_{1}\left(u_{0}, w\left(u_{0} ; \alpha, \beta\right)\right)-\sigma\right]
$$

where $n<0$ and $(\alpha, \beta) \in U$. We want the left side $\geqq 0$, and this will hold if $(L)$ holds. Relation (2.23) can be obtained from (2.9) and (2.10). We leave the details to the reader.

We shall next show that condition $(L)$, along with our other assumptions on $F$, actually characterizes the ordering principle $C\left(P_{1}\right) \subseteq C\left(P_{0}\right)$.

Theorem 2.9. Suppose that the system (1.1) satisfies (2.1) and (2.7) in $U$. Then $C\left(P_{1}\right) \subseteq C\left(P_{0}\right)$ for every $P_{1} \in C\left(P_{0}\right), P_{0}, P_{1} \in U$, if and only if condition $(L)$ holds in $U$.

Proof. We have already proved that condition $(L)$ is sufficient. Now suppose that the ordering condition holds and condition $(L)$ is violated. Then from part (1) of Lemma 2.5, there is some point $P^{\prime} \in U$ for which $\lambda_{1}\left(P_{0}\right)=\sigma\left(P^{\prime}, P_{0}\right)$. Since $\sigma$ decreases along shock curves (see the proof of Theorem 2.2), we can find a point $P \in U$ such that $\lambda_{1}\left(P_{0}\right)>\sigma\left(P, P_{0}\right)$. As before, using (2.21), it follows that $\alpha \neq 0$. Also, the geometry shows that $\alpha>0$ is impossible (otherwise $r_{1}(P)=n r_{1}\left(P_{0}\right)+$ $m\left(P-P_{0}\right)$ with $\left.n<0\right)$ so that $\alpha<0$. But since $\alpha=\theta^{\prime}(\tau)$, there exist $\tau_{1}, \tau_{2}$, with $\tau_{2}>\tau_{1}$, for which $\theta\left(\tau_{1}\right)>\theta\left(\tau_{2}\right)$. This implies that $\phi_{\theta\left(\tau_{1}\right)} P$ is further along the wave curve $v=w(u ; P)$ than $\phi_{\theta\left(\tau_{2}\right)} P$, so that the shock curve $v=s\left(u ; \phi_{\tau_{1}}{ }^{\prime} P_{0}\right)$ crosses the shock curve $v=s\left(u ; \phi_{\tau_{2}} P_{0}\right)$. This contradicts the ordering principle and the proof is complete.

We end this section by remarking that the ordering principle will hold if we have a uniqueness theorem for Riemann problems in $C\left(P_{0}\right)$. To see this, suppose 
that $P_{1}=\left(u_{1}, v_{1}\right)$ is on the wave curve $v=w\left(u ; P_{0}\right)$ and that the shock curve $v=s\left(u ; P_{1}\right)$ meets the shock curve $v=s\left(u ; P_{0}\right)$ at $P_{2}=\left(u_{2}, v_{2}\right)$, see Figure 3 . Then the Riemann problem for (1.1) with initial data

has the two distinct solutions

$$
(u(x, 0), v(x, 0))= \begin{cases}\left(u_{0}, v_{0}\right), & x<0 \\ \left(u_{2}, v_{2}\right), & x>0\end{cases}
$$
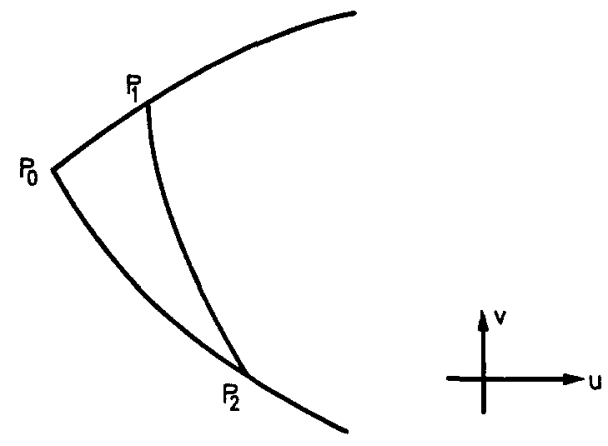

Fig. 3

(a) $\left(u_{0}, v_{0}\right),\left(u_{2}, v_{2}\right)$; i.e., two constant states separated by a 2 -shock, and

(b) the three constant states $\left(u_{0}, v_{0}\right),\left(u_{1}, v_{1}\right),\left(u_{2}, v_{2}\right)$ where $\left(u_{1}, v_{1}\right)$ is connected to $\left(u_{0}, v_{0}\right)$ by a 1-rarefaction wave, and $\left(u_{2}, v_{2}\right)$ is connected to $\left(u_{1}, v_{1}\right)$ by a 2 -shock.

Thus, anticipating our work in the next section, we can say that in this case uniqueness for Riemann problems implies existence for more general data. We note that it has been proposed in [9] that the shock conditions (2.16) and $(L)$ should be sufficient to isolate a unique solution (possibly the physically relevent one). We have given further evidence for this conjecture by showing that in view of Theorem 2.9 , condition $(L)$ must necessarily hold if there is a unique solution to the Cauchy problem (1.1), (1.2). In view of its importance, we state this formally as a theorem:

Theorem 2.10. Let the system (1.1) satisfy (2.1) and (2.7) in U. If the Cauchy problem (1.1), (1.2) has a unique solution in $U$, then condition $(L)$ holds in $U$.

We remark that similar considerations using shock curves of the first characteristic family show that uniqueness implies that the following condition must also hold in $U$ :

For each $P_{0} \in U$, if $v=s_{1}\left(u ; P_{0}\right)$ is the shock curve of the first characteristic $\left(L^{\prime}\right)$ family through $P_{0}$, and $(u, v) \in U$, then $\sigma_{1}\left(u, v ; P_{0}\right)<\lambda_{2}(u, v)$ where $\sigma_{1}$ denotes the corresponding shock speed.

\section{The Existence Theorem}

We shall now sketch a proof of the existence theorem. The results of Section 2 allow us almost completely to carry over the argument of [7]. The main difficulty is that we cannot show, as in [7], that our approximating solutions are of locally 
bounded variation in the sense of TONELLI-CESARI with respect to the $(x-t)$ coordinates. This is true because we do not necessarily have $\lambda_{2}>0>\lambda_{1}$ but merely $\lambda_{2}>\lambda_{1}$.

We require that our initial data $\left(u_{0}(x), v_{0}(x)\right)$ be bounded ${ }^{\star}$ and measurable and satisfy the following condition in $U$ :

$$
\left(u_{0}\left(x_{2}\right), v_{0}\left(x_{2}\right)\right) \in C\left(u_{0}\left(x_{1}\right), v_{0}\left(x_{1}\right)\right), \quad x_{1}<x_{2} .
$$

Note that Theorem 2.6 implies that the relation $\left(u_{0}\left(x_{2}\right), v_{0}\left(x_{2}\right)\right) \in C\left(u_{0}\left(x_{1}\right), v_{0}\left(x_{1}\right)\right)$ is transitive, thereby making it somewhat easier to check $(C)$.

Theorem 3.1. Let the system (1.1) satisfy (2.1), (2.7) and condition $(L)$ in $U \subseteq E^{2}$. Let the initial data (1.2) be bounded and measurable and satisfy condition $(C)$ in $U$. Then the Cauchy problem (1.1), (1.2) has a solution.

The method of proof is to approximate the initial data with step data $\left(u_{n}^{0}(x)\right.$, $\left.v_{n}^{0}(x)\right)$ such that condition $(C)$ is maintained, and then to solve the corresponding initial-value problems as in [7] to obtain solutions $\left(u_{n}(x, t), v_{n}(x, t)\right), t \geqq 0$. The functions $u_{n}(t, x)$ are uniformly bounded, for at each fixed $t_{0}$

$$
\lim _{x \rightarrow \pm \infty} u_{n}\left(x, t_{0}\right)=u_{0}( \pm \infty)
$$

and $u_{n}\left(t_{0}, x\right)$ is monotonic in $x$. Let $v_{+}$and $v_{-}$be upper and lower bounds for $v_{0}(x)$. Then from our previous paper [7] we know that $\left(u_{n}(t, x), v_{n}(t, x)\right)$ is contained in the compact region $B$ bounded by

(a) the shock curve with $\left(u_{0}(-\infty), v_{-}\right)$on the left

(b) the shock curve with $\left(u_{0}(+\infty), v_{+}\right)$on the right

(c) the line $u=u_{0}(+\infty)$

(d) the line $u=u_{0}(-\infty)$.

Hence $v_{n}(x, t)$ is uniformly bounded, independent of $n$. Let $k$ be an upper bound for the slopes of the wave and shock curves in $B$.

Now note that if $x=x(s), t=t(s), a<s<b$, is a curve which crosses both waves and shocks, in our solutions, from left to right as $s$ increases, then $u_{n}(x(s)$, $t(s)$ ) is a non-decreasing function of $s$. Furthermore, by definition of $k$,

$$
\left|v_{n}\left(P_{1}\right)-v_{n}\left(P_{2}\right)\right| \leqq k\left|u_{n}\left(P_{1}\right)-u_{n}\left(P_{2}\right)\right|
$$

where $P_{i}=\left(x\left(s_{i}\right), t\left(s_{i}\right)\right), i=1,2$. Hence $v_{n}(x(s), t(s))$ is of bounded variation, where the bound on the variation is independent of $n$.

Let

and define

$$
\alpha>\sup \left\{\lambda_{2}(u, v):(u, v) \in B\right\}
$$

$$
\bar{u}_{n}(x, t)=u_{n}(x+\alpha t, t), \quad \bar{v}_{n}(x, t)=v_{n}(x+\alpha t, t) .
$$

For each fixed $t_{0}$, the $x$-variation of $\vec{v}_{n}\left(x, t_{0}\right)$ is

$$
V_{x}\left[\bar{v}_{n}\left(x, t_{0}\right)\right]=V_{x}\left[v_{n}\left(x+\alpha t_{0}, t_{0}\right)\right]=V_{x}\left[v_{n}\left(x, t_{0}\right)\right],
$$

* Actually, as in [7], we need only require that one of the two functions be bounded. As things are set up here, we need only require that $u_{0}(x)$ be bounded. 
and hence is bounded. Similarly, for fixed $x_{0}$, the $t$-variation of $\bar{v}_{n}\left(t, x_{0}\right)$ is

$$
V_{t}\left[\bar{v}_{n}\left(x_{0}, t\right)\right]=V_{t}\left[v_{n}\left(x_{0}+\alpha t, t\right)\right],
$$

which is the variation of $v_{n}(x, t)$ along the line $x=x_{0}+\alpha t$. Since this line crosses both shocks and waves from left to right, by definition of $\alpha$, we see that $V_{t}\left[\bar{v}_{n}\left(x_{0}, t\right)\right]$ is also bounded independent of $n$. Therefore $\bar{v}_{n}(x, t)$ is of uniformly bounded TONELLI-CESARI variation on each compact subset of $t \geqq 0$, (see [7]). In an analogous fashion, $\bar{u}_{n}(x, t)$ is of uniformly bounded Tonelli-CeSARI variation on each compact subset of $t \geqq 0$.

From [3] and [6], these functions are compact in the sense of $L_{1}$-convergence on compacta in $t \geqq 0$. Thus we get a subsequence, call it $\left(\bar{u}_{n}(x, t), \bar{v}_{n}(x, t)\right)$ again, such that

$$
\left(\bar{u}_{n}(x, t), \bar{v}_{n}(x, t)\right) \rightarrow(\bar{u}(x, t), \bar{v}(x, t))
$$

in the sense of $L_{1}$-convergence on compacta in $t \geqq 0$. If we now let

$$
u(x, t)=\bar{u}(x-\alpha t, t), \quad v(x, t)=\bar{v}(x-\alpha t, t),
$$

then by a change of variables in the multiple integral we have

$$
\begin{aligned}
\iint_{t \geqq 0} & {\left[\left|u(x, t)-u_{n}(x, t)\right|+\left|v(x, t)-v_{n}(x, t)\right|\right] d x d t } \\
& =\iint_{t \geqq 0}\left[\left|\bar{u}(x-\alpha t, t)-\vec{u}_{n}(x-\alpha t, t)\right|+\left|\bar{v}(x-\alpha t, t)-\bar{v}_{n}(x-\alpha t, t)\right|\right] d x d t \\
& =\iint_{t \geqq 0}\left[\left|\bar{u}(x, t)-\bar{u}_{n}(x, t)\right|+\left|\bar{v}(x, t)-\bar{v}_{n}(x, t)\right|\right] d x d t
\end{aligned}
$$

Hence

$$
\left(u_{n}(x, t), v_{n}(x, t)\right) \rightarrow(u(x, t), v(x, t))
$$

in the sense of $L_{1}$ convergence on compacta in $t \geqq 0$. The fact that $(u(x, t), v(x, t))$ is a solution of our original problem (1.1), (1.2) now follows as in [7].

\section{Existence Theorem Using the Glimm Difference Scheme}

Let $f$ and $g$ satisfy the same hypotheses as before, namely (2.2), (2.7) and condition $(L)$ in $U \subseteq E^{2}$, and let the initial data $U_{0}(x)=\left(u_{0}(x), v_{0}(x)\right)$ be bounded and measurable and satisfy condition $(C)$ in $U$. We shall show that we can obtain a solution to the problem (1.1), (1.2) using the method of GLIMM [4].

We need to recall briefly the definition of the GLIMM difference scheme. Let $B$ be the compact region defined in Section 3. Choose mesh lengths $r$ and $s$ such that

$$
\sup \left\{\left|\lambda_{i}(u, v)\right|:(u, v) \in B, i=1,2\right\}<r / s,
$$

and hold $r / s$ fixed so that $s$ is a function of $r$. Let

and let

$$
Y=\{(m, n): m, n \text { integers, } m+n \equiv 0(2), n \geqq 0\},
$$

$$
A=\prod\{[(m-1) r,(m+1) r] \times\{n s\}:(m, n) \in Y\} .
$$

Give each factor of $A$ a measure $1 / 2 r$ times its Lebesgue measure and let $A$ have the product measure. Choose a point $a=\left\{a_{m, n}\right\}$ in $A$ which is the random choice 
of our mesh points in $t \geqq 0$. For each $(m, n) \in Y$, let $V(x, t)=(u(x, t), v(x, t))$ be a solution of $(1.1)$ in the rectangle $(m-1) r \leqq x \leqq(m+1) r, n s \leqq t<(n+1) s$, having initial values

$$
\left(u_{0}(x), v_{0}(x)\right)= \begin{cases}U_{0}\left(a_{m, n}\right), & (m-1) r \leqq x<m r \\ U_{0}\left(a_{m+1, n}\right), & m r \leqq x<(m+1) r\end{cases}
$$

By our previous results, the solution exists since the data satisfies condition $(C)$. Also, in view of Theorem 2.9, the solution also satisfies condition $(C)$ on $t=(n+1) s$. We set

$$
U(x, t)=V(x, t), \quad(m-1) r \leqq x \leqq m r, \quad n s \leqq t \leqq(n+1) s,
$$

and then $U(x, t)$ is defined on the line $t=(n+1) s$. Moreover, $U(x, t)$ restricted to the mesh points $a_{m, n+1}$ also satisfies condition $(C)$, and hence the difference scheme is defined inductively on all the mesh points (compare with [4], p. 705). Hence

$$
U_{a, r}=U(x, t)
$$

is defined by this device on $t \geqq 0$. Next, from our work in Section 3 , there exist constants $k_{i}$ such that for each $t_{0} \geqq 0$, if $x_{1} \geqq x_{2}$

$$
\begin{aligned}
\left|v\left(x_{1}, t_{0}\right)-v\left(x_{2}, t_{0}\right)\right| & \leqq k_{1}\left|u\left(x_{1}, t_{0}\right)-u\left(x_{2}, t_{0}\right)\right| \\
& =k_{1}\left[u\left(x_{1}, t_{0}\right)-u\left(x_{2}, t_{0}\right)\right] \leqq k_{2} \text { tot. var. } U_{0}(x),
\end{aligned}
$$

so that for each $t \geqq 0$,

$$
\text { Tot. var. } U(t, x) \leqq k_{3} \text { Tot. var. } U_{0}(x) .
$$

It now follows as in [4], pp. 711-714, that there exists a set of measure zero $N \subset A$, such that if $a \in A \sim N$ then a sequence $U_{a, r_{i}}$ converges, as $r_{i} \rightarrow 0$, to a solution of (1.1), (1.2).

We therefore can obtain a solution to our problem using the GLIMM difference scheme. However, since we are unaware of any uniqueness theorem for systems which is applicable here, we do not know whether the solution constructed in Section 3 agrees with the solution constructed here. We conjecture an affirmative answer to this question.

\section{Concluding Remarks}

We first note, just as in [7], that the functions $\bar{u}(x, t)$ and $\bar{v}(x, t)$ defined in Section 3 are monotonic with respect to $x$ and $t$ respectively. It then follows, as we observed in [7], that these functions are continuous almost everywhere in $t \geqq 0$. Therefore the solution $(u(x, t), v(x, t))$ constructed in Section 3 is continuous almost everywhere in $t \geqq 0$.

Next we would like to make an observation concerning condition (2.7). It might appear that this condition, being a type of convexity condition on the mapping $F$, would be implied by the definiteness of the Hessians of $f$ and $g$. We shall now show that this is false. Let $\mathscr{I}$ and $\mathscr{J}$ be the Hessians of $f$ and $g$, respectively. From (2.5) it follows that $\mathscr{I}<0$ and $\mathscr{J}<0$ implies that $l_{1} d^{2} F\left(r_{2}, r_{2}\right)<0$, and 
$\mathscr{J}>0, \mathscr{I}<0$ implies that $l_{2} d^{2} F\left(r_{2}, r_{2}\right)<0$. Thus we must only consider the cases $\mathscr{I}>0, \mathscr{J}>0$ and $\mathscr{I}<0, \mathscr{J}>0$. Let $(0,0) \in U$ and consider the smooth functions $f_{i}$ and $g_{i}$ :

$$
\begin{array}{ll}
f_{1}(u, v)=u-v+\frac{1}{6} u^{2}+\frac{1}{12} v^{2}, & f_{2}(u, v)=u-v-\frac{1}{3} u^{2}-\frac{1}{6} v^{2}, \\
g_{1}(u, v)=-2 u+\frac{11}{20} u^{2}+\frac{1}{20} v^{2}, & g_{2}(u, v)=-2 u+\frac{1}{12} u^{2}+\frac{17}{48} v^{2} .
\end{array}
$$

Let $F_{i}=\left(f_{i}, g_{i}\right)$, and let $\mathscr{I}_{i}$ and $\mathscr{J}_{i}$ be the Hessians of $f_{i}$ and $g_{i}$ respectively, $i=1,2$. At the point $(0,0)$, each matrix $d F_{i}$ is the same:

$$
\left[\begin{array}{rr}
1 & -1 \\
-2 & 0
\end{array}\right]
$$

This matrix has eigenvalues $\lambda_{1}=-1, \lambda_{2}=2$ with right and left eigenvectors given by

At $(0,0)$,

$$
\begin{array}{ll}
r_{1}=(1,2)^{t}, & r_{2}=(-1,1)^{t}, \\
l_{1}=(1,1), & l_{2}=(-2,1) .
\end{array}
$$

$$
d^{2} F_{1}\left(r_{1}, r_{1}\right)=(-2,3)^{t}, \quad d^{2} F_{1}\left(r_{2}, r_{2}\right)=(-1,3 / 4)^{t}
$$

so that $l_{i} d^{2} F_{1}\left(r_{1}, r_{1}\right)>0, i=1,2, l_{2} d^{2} F_{1}\left(r_{2}, r_{2}\right)>0$, but $l_{1} d^{2} F\left(r_{2}, r_{2}\right)<0$. Furthermore it is easy to check that at $(0,0), \mathscr{I}_{1}>0$ and $\mathscr{J}_{1}>0$. Hence by continuity, we see that these inequalities are true in a neighborhood of $(0,0)$. A similar argument using $f_{2}$ and $g_{2}$ shows that $\mathscr{I}_{2}<0$ and $\mathscr{J}_{2}>0$ do not imply (2.7). We remark that it is not difficult to show that if $\mathscr{J}>0$, then $\mathscr{I}>0$ implies that $l_{1} d^{2} F\left(r_{2}, r_{2}\right)>0$, while $\mathscr{I}<0$ implies that $l_{2} d^{2} F\left(r_{1}, r_{1}\right)>0$. Here we are of course assumming that the $r_{i}$ and $l_{i}$ are normalized by (2.4) and $l_{i} r_{i}>0, i=1,2$.

We would also like to point out that we do not need both conditions (2.6) in order to prove an existence theorem using either the method of Section 3 or the method of Section 4. For example, we only used $l_{2} d^{2} F\left(r_{1}, r_{1}\right)>0$ in order to prove that the wave curve does not escape. If the wave curve did happen to escape, this would cause us no difficulty. That is, we can still solve the Riemann problems for (1.1) with initial data satisfying $(C)$, by a 1-rarefaction wave and a 2-shock wave, and the solutions will have the same properties as before.

Finally, in view of Theorems 2.9 and 2.10 , it would be very interesting to know whether condition $(L)$ is always valid globally.

\section{Appendix}

Consider a hyperbolic genuinely non-linear system of quasi-linear equations in a single space variable of the form

$$
u_{t}+F(u)_{x}=0
$$

where $u$ and $F$ are vector functions of dimension $n$. In this section we shall demonstrate a relation between the $j$-waves produced in the interaction of two $k$-shocks and the second derivative $d^{2} F$. Our notation and terminology is that of GLIMM [4]. 
We assume that we can find a local coordinate system $w_{1}, w_{2}, \ldots, w_{n}$ defined in an open set $U \subset E^{n}$ which consists of Riemann invariants. This means that the functions $w_{j}$ satisfy

$$
d w_{j}(u) r_{j}(u)=\delta_{i j}, \quad u \in U, \quad i, j=1,2, \ldots, n,
$$

where the $r_{j}(u)$ are right eigenvectors of $A=d F(u)$, for the eigenvalue $\lambda_{i}(u)$.

Let $v_{1}, v_{m}$ and $v_{r}$ be three constant states such that $v_{m}$ is connected to $v_{1}$ on the right by a $k$-shock of magnitude $\delta$, and $v_{r}$ is connected to $v_{m}$ on the right by a $k$-shock of magnitude $\gamma$. From the LAX shock conditions ([9], eqs. (7.1)), we see that these two shocks must intersect. At the time of intersection, we have to solve a Riemann problem with $v_{1}$ on the left and $v_{r}$ on the right. If $v_{1}, v_{m}$ and $v_{r}$ all lie in $U$, and $U$ is sufficiently small, then the solution of this problem exists by Theorem (9.1) of [9], and consists of constant values $v_{1}=v_{0}, v_{1}, \ldots, v_{n}=v_{r}$, separated by shocks or rarefaction waves. The magnitude of the $j$-wave produced in this interaction is $\varepsilon_{j}$ where $\varepsilon_{j}$ is defined by $\varepsilon_{j}=w_{j}\left(v_{j}\right)-w_{j}\left(v_{j-1}\right)$. If $\varepsilon_{j}>0$ then the $j$-wave produced in this interaction is a rarefaction wave, while if $\varepsilon_{j}<0$, the $j$-wave is a shock wave. Our main goal in this section is to prove that

$$
\operatorname{sgn} \varepsilon_{j}=\operatorname{sgn} l_{j} d^{2} F\left(r_{k}, r_{k}\right)
$$

where $l_{j}$ is the left eigenvector of $A$ for the eigenvalue $\lambda_{j}$, normalized by $l_{j} r_{j}=1$, $i, j=1,2, \ldots, n$, provided that $l_{j} d^{2} F\left(r_{k}, r_{k}\right) \neq 0$. Our first step in this direction is the following lemma which extends a result in [9].

Lemma 1. If $v_{j}$ is connected to $v_{j-1}$ by a j-shock then

$$
\begin{aligned}
v_{j}= & v_{j-1}+\varepsilon_{j} r_{j}+1 / 2 \varepsilon_{j}^{2} d r_{j}\left(r_{j}\right) \\
& +1 / 6 \varepsilon_{j}^{3}\left[d^{2} r_{j}\left(r_{j}, r_{j}\right)-\sum_{k \neq j} \frac{l_{k} \dot{r}_{j}}{\left(\lambda_{k}-\lambda_{j}\right)} r_{k}+k_{j} r_{j}\right]+O\left(\varepsilon_{j}^{4}\right),
\end{aligned}
$$

where $k_{j}$ is a constant independent of $\varepsilon_{j}$ and the $r_{j}$ 's are all evaluated at $v_{j-1}$.

Proof. We need only calculate the coefficient of $\varepsilon_{j}^{3} / 6$, the other terms being given in [9]. Let $u_{j}\left(\varepsilon_{j}\right)$ be the curve of states which can be connected to $v_{j-1}$ by a $j$-shock on the right. Take the equations $s\left(u-u_{1}\right)=F(u)-F\left(u_{1}\right), A r_{j}=\lambda_{j} r_{j}$ with $u=u_{j}\left(\varepsilon_{j}\right)$, differentiate the first three times with respect to $\varepsilon_{j}$, the second twice with respect to $\varepsilon_{j}$, and set $\varepsilon_{j}=0$. This gives

and

$$
\ddot{A} r_{j}+2 \dot{A} \dot{r}_{j}+A \dddot{u}_{j}=\lambda_{j} \dddot{u}_{j}+3 \dot{r}_{j} / 2+3 \ddot{s} r_{j}
$$

$$
\ddot{A} r_{j}+2 \dot{A} \dot{r}_{j}+A \ddot{r}_{j}=\lambda_{j} \ddot{r}_{j}+2 \dot{r}_{j}+\ddot{\lambda}_{j} r_{j}
$$

where we have used the normalizations in [9]. Subtracting, we get

$$
A\left(\ddot{r}_{j}-\dddot{u}_{j}\right)=\lambda_{j}\left(\ddot{r}_{j}-\dddot{u}_{j}\right)+\dot{r}_{j} / 2+\left(\ddot{\lambda}_{j}-3 \ddot{s}\right) r_{j} .
$$

If we multiply this equation on the left by $l_{j}$, we get $l_{j} \dot{r}_{j} / 2+\ddot{\lambda}_{j}-3 \ddot{s}=0$ so that (5) can be written

$$
\left(A-\lambda_{j} I\right)\left(\ddot{r}_{j}-\dddot{u}_{j}\right)=\frac{1}{2}\left[\dot{r}_{j}-\left(l_{j} \dot{r}_{j}\right) r_{j}\right] \text {. }
$$


If we define an operator $L$ by $L r_{i}=\left(\lambda_{i}-\lambda_{j}\right)^{-1} r_{i}$ if $i \neq j, L r_{j}=r_{j}$, then $L$ is a pseudo-inverse for $\left(A-\lambda_{j} I\right)$ so that we can write

$$
\ddot{r}_{j}-\dddot{u}_{j}=\frac{1}{2} L\left[\dot{r}_{j}-\left(l_{j} \dot{r}_{j}\right) r_{j}\right]+c_{j} r_{j}
$$

where $c_{j}$ is some constant. Since $\dot{r}_{j}-\left(l_{j} \dot{r}_{j}\right) r_{j}=\sum_{k \neq j}\left(l_{k} \dot{r}_{j}\right) r_{k}$, we get from (6) that

$$
\ddot{r}_{j}-\dddot{u}_{j}=\frac{1}{2} \sum_{k \neq j} l_{k} \dot{r}_{j}\left(\lambda_{k}-\lambda_{j}\right)^{-1} r_{k}+c_{j} r_{j} .
$$

The next lemma shows precisely how the Riemann invariants change along shock curves.

Lemma 2. If $i \neq j$, then

$$
w_{i}\left(v_{j}\right)-w_{i}\left(v_{j-1}\right)=-\frac{\alpha_{j} l_{i} \dot{r}_{j}}{12\left(\lambda_{i}-\lambda_{j}\right)} \varepsilon_{j}^{3}+O\left(\varepsilon_{j}^{4}\right),
$$

where $\alpha_{j}=1$ if $v_{j}$ is connected to $v_{j-1}$ by a j-shock, $\alpha_{j}=0$ if $v_{j}$ is connected to $v_{j-1}$ by a j-rarefaction wave and $l_{i} \dot{r}_{j}\left(\lambda_{i}-\lambda_{j}\right)^{-1}$ is evaluated at $v_{j-1}$.

Proof. If $v_{j}$ is connected to $v_{j-1}$ by a $j$-rarefaction wave, then by definition of $w_{i}, w_{i}\left(v_{j}\right)-w_{i}\left(v_{j-1}\right)=\delta_{i j} \varepsilon_{j}=0$. On the other hand, if $v_{j}$ is connected to $v_{j-1}$ by a $j$-shock, then (4) holds, so by a Taylor expansion about $v_{j-1}$ we get

$$
\begin{aligned}
w_{i}\left(v_{j}\right)-w_{i}\left(v_{j-1}\right)= & \varepsilon_{j} d w_{i} r_{j}+\frac{\varepsilon_{j}^{2}}{2}\left[d^{2} w_{i}\left(r_{j}, r_{j}\right)+d w_{i} \dot{r}_{j}\right] \\
& +\frac{\varepsilon_{j}^{3}}{6}\left[d^{3} w_{i}\left(r_{j}, r_{j}, r_{j}\right)+3 d^{2} w_{i}\left(r_{j}, \dot{r}_{j}\right)+d w_{i} \beta_{j}\right]+O\left(\varepsilon_{j}^{4}\right)
\end{aligned}
$$

where

$$
\beta_{j}=d^{2} r_{j}\left(r_{j}, \dot{r}_{j}\right)+d r_{j} \dot{r}_{j}-\frac{1}{2} \sum_{k \neq j} l_{k} \dot{r}_{j}\left(\lambda_{k}-\lambda_{j}\right)^{-1} r_{k}+k_{j} r_{j}
$$

and the $r_{j}$ 's are all evaluated at $v_{j-1}$. Next, we consider (1), valid for all $u \in U$, specialize to $u=u_{j}\left(\varepsilon_{j}\right)$, differentiate this twice with respect to $\varepsilon_{j}$ and set $\varepsilon_{j}=0$. This gives

and

$$
d^{2} w_{i}\left(r_{j}, r_{j}\right)+d w_{i}\left(\dot{r}_{j}\right)=0
$$

$$
d^{3} w_{i}\left(r_{j}, r_{j}, r_{j}\right)+3 d^{2} w_{i}\left(r_{j}, \dot{r}_{j}\right)+d w_{i} \ddot{r}_{j}=0
$$

Therefore (8) becomes

$$
w_{i}\left(v_{j}\right)-w_{i}\left(v_{j-1}\right)=\frac{\varepsilon_{j}^{3}}{6}\left[d w_{i}\left(\beta_{j}-\ddot{r}_{j}\right)\right]+O\left(\varepsilon_{j}^{4}\right)=-\frac{\varepsilon_{j}^{3}}{12} \frac{l_{i} \dot{r}_{j}}{\left(\lambda_{i}-\lambda_{j}\right)}+O\left(\varepsilon_{j}^{4}\right)
$$

in view of (2).

With these lemmas in mind, we can proceed to our main result. Since $w_{i}\left(v_{i}\right)-$ $w_{i}\left(v_{i-1}\right)=\varepsilon_{i}$, we get, by summing (7) on $j$,

$$
w_{i}\left(v_{r}\right)-w_{i}\left(v_{1}\right)=\varepsilon_{i}-\frac{1}{12} \sum_{j \neq i} \varepsilon_{j}^{3} \alpha_{j} \frac{l_{i} \dot{r}_{j}}{\left(\lambda_{i}-\lambda_{j}\right)}+O\left(|\varepsilon|^{4}\right) .
$$


From equation (2.7) in [4], we have for $i=1,2, \ldots, n$,

$$
w_{i}\left(v_{i}\right)-w_{i}\left(v_{i-1}\right)=\varepsilon_{i}=\gamma_{i}+\delta_{i}+O\left([|\gamma|+|\delta|]^{3}\right) .
$$

Now since $\gamma_{k}+\delta_{k}<0$, we see that $\varepsilon_{k}<0$ for small $|\gamma|+|\delta|$, so that $v_{k}$ is connected to $v_{k-1}$ by a $k$-shock and thus $\alpha_{k}=1$ in (9). Moreover if $i \neq k$, then $\gamma_{i}=\delta_{i}=0$, so that (9) becomes, for $i \neq k$,

$$
\varepsilon_{i}=w_{i}\left(v_{r}\right)-w_{i}\left(v_{1}\right)+\frac{\left(\gamma_{k}+\delta_{k}\right)^{3}}{12} \frac{l_{i} \dot{r}_{k}}{\left(\lambda_{i}-\lambda_{k}\right)}+O\left([|\gamma|+|\delta|]^{4}\right) .
$$

We shall next exhibit a relation between $l_{i} \dot{r}_{k}$ and $d^{2} F$. The basis of this relation is obtained by differentiating the equation $A r_{k}=\lambda_{k} r_{k}$ in the direction $r_{k}$ :

$$
d \lambda_{k}\left(r_{k}\right)\left(r_{k}\right)+\lambda_{k} d r_{k}\left(r_{k}\right)=d A\left(r_{k}\right) r_{k}+A d r_{k}\left(r_{k}\right) .
$$

If we multiply this equation on the left by $l_{i}, i \neq k$, we obtain

$$
l_{i} d r_{k}\left(r_{k}\right)=\frac{1}{\left(\lambda_{k}-\lambda_{i}\right)}\left[l_{i} d A\left(r_{k}\right) r_{k}\right]=\frac{1}{\left(\lambda_{k}-\lambda_{i}\right)} l_{i} d^{2} F\left(r_{k}, r_{k}\right) .
$$

Substituting in (10), we get for $i \neq k$,

$$
\varepsilon_{i}=w_{i}\left(v_{r}\right)-w_{i}\left(v_{1}\right)-\frac{\left(\gamma_{k}+\delta_{k}\right)^{3}}{12} \frac{l_{i} d^{2} F\left(r_{k}, r_{k}\right)}{\left(\lambda_{i}-\lambda_{k}\right)^{2}}+O\left([|\gamma|+|\delta|]^{4}\right)
$$

where $\left(\lambda_{i}-\lambda_{k}\right)^{-2} l_{i} d^{2} F\left(r_{k}, r_{k}\right)$ is evaluated at $v_{k-1}$. Next from Lemma 2, if $i \neq k$,

and

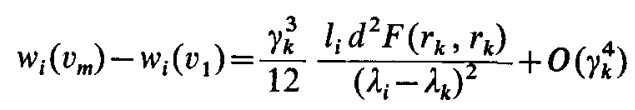

$$
w_{i}\left(v_{r}\right)-w_{i}\left(v_{m}\right)=\frac{\delta_{k}^{3}}{12} \frac{l_{i} d^{2} F\left(r_{k}, r_{k}\right)}{\left(\lambda_{i}-\lambda_{k}\right)^{2}}+O\left(\delta_{k}^{4}\right) .
$$

If we substitute in (13) and use the fact that $v_{k-1}$ is a $C^{2}$ function of $v_{1}$ ([9], p. 563), we get

$$
\varepsilon_{i}=-\frac{\gamma_{k}^{2} \delta_{k}+\gamma_{k} \delta_{k}}{4\left(\lambda_{i}-\lambda_{k}\right)^{2}} l_{i} d^{2} F\left(r_{k}, r_{k}\right)+O\left([|\gamma|+|\delta|]^{4}\right), \quad i \neq k ;
$$

and from this we see that (3) holds for small $|\gamma|+|\delta|$ provided that $l_{i} d^{2} F\left(r_{k}, r_{k}\right) \neq 0$. We have thus proved the following theorem.

Theorem 1. Let the system (1) be hyperbolic, genuinely non-linear, and admit Riemann invariants in an open set $U \subset E^{n}$. Let $r_{j}(u), l_{j}(u)$ denote the left and right eigenvectors of $d F(u)$ for the eigenvalue $\lambda_{j}(u)$, normalized by $d \lambda_{j}(u) r_{j}(u)>0$, $l_{j}(u) r_{j}(u)>0, j=1,2, \ldots, n$. Then the interaction of two sufficiently weak $k$-shocks produces a $j$-shock, $j \neq k$, at $u \in U$ if $l_{j}(u) d^{2} F\left(r_{k}(u), r_{k}(u)\right)<0$, and this interaction produces a j-rarefaction wave, $j \neq k$, at $u \in U$ if $l_{j}(u) d^{2} F\left(r_{k}(u), r_{k}(u)\right)>0$.

In the case where $l_{j}(u) d^{2} F\left(r_{k}(u), r_{k}(u)\right)=0$, one can obtain the sign of $\varepsilon_{j}$ by calculating the higher order terms in (4) and obtaining an analogue of (7). We shall not pursue these matters here. We note that in the course of the proof we 
have shown that the $k$ wave produced in the interaction of two sufficiently weak $k$-shocks will be a $k$-shock wave.

We shall next demonstrate a relation between $d^{2} F$ and the condition of genuine non-linearity. To this end, multiply (11) on the left by $l_{k}$ and get $d \lambda_{k}\left(r_{k}\right)=$ $l_{k} d A\left(r_{k}\right) r_{k}=l_{k} d^{2} F\left(r_{k}, r_{k}\right)$. This proves the following theorem.

Theorem 2. The system (1) is genuinely non-linear in the $k$-th characteristic field, $1 \leqq k \leqq n$ at $u \in E$ if and only if $l_{k}(u) d^{2} F\left(r_{k}(u), r_{k}(u)\right) \neq 0$.

We remark that for certain systems of equations we have the equalities

$$
l_{j}(u) d^{2} F\left(r_{k}(u), r_{k}(u)\right)=l_{j}(u) d^{2} F\left(r_{k}(u), r_{k}(u)\right)
$$

so that the condition of genuine non-linearity is equivalent to a shock interaction condition. This is true, for example, for the system $u_{t}+f(v)_{x}=0, v_{t}+g(u)_{x}=0$ considered in [7].

Now we shall specialize to the case $n=2$ where our results can be formulated in terms of the classical Riemann invariants of the system. Thus consider the system (1.1) with the notations in $\S 1$. The left eigenvectors of $A$ can be taken as $L_{1}=\left(r_{u}, r_{v}\right), L_{2}=\left(s_{u}, s_{v}\right)$, and upon multiplying (1.1) by these we obtain the Riemann formulation $r_{t}+\lambda_{1}(r, s) r_{x}=0, s_{t}+\lambda_{2}(r, s) s_{x}=0$. If $\tilde{R}_{1}=\left(s_{v},-s_{u}\right)^{t}$, $\tilde{R}_{2}=\left(-r_{v} r_{w}\right)^{t}$, then corresponding right eigenvectors with the normalization $L_{i} R_{i}=1, i=1,2$, are given by $R_{i}=k \tilde{R}_{i}, i=1,2$, where $k=\left(r_{u} s_{v}-r_{v} s_{u}\right)^{-1}$. Referring to (12), we have

$$
\begin{aligned}
\left(\lambda_{2}-\lambda_{1}\right)^{-1} L_{1} d^{2} F\left(R_{2}, R_{2}\right) & =\left(\lambda_{2}-\lambda_{1}\right)^{-1} k^{2} L_{1} d^{2} F\left(R_{2}, R_{2}\right)=k^{2} L_{1} d \tilde{R}_{2}\left(\tilde{R}_{2}\right) \\
= & k^{2}\left(r_{u}, r_{v}\right)\left(r_{v} r_{v u}-r_{u} r_{v v},-r_{v} r_{u u}+r_{u} r_{u v}\right)^{t}=-R_{2}^{t} \mathscr{R} R_{2},
\end{aligned}
$$

where

$$
\mathscr{R}=\left(\begin{array}{ll}
r_{u} & r_{u v} \\
r_{u v} & v v
\end{array}\right)
$$

Therefore

$$
L_{1} d^{2} F\left(R_{2}, R_{2}\right)=\left(\lambda_{1}-\lambda_{2}\right) R_{2}^{t} \mathscr{R} R_{2}
$$

and similarly

$$
L_{2} d^{2} F\left(R_{1}, R_{1}\right)=\left(\lambda_{2}-\lambda_{1}\right) R_{1}^{t} \mathscr{S} R_{1}
$$

where

$$
\mathscr{P}=\left(\begin{array}{ll}
s_{u u} & s_{u v} \\
s_{u v} & s_{v v}
\end{array}\right)
$$

Finally, we recall the shock interaction condition introduced in [5] which states that the interaction of two shocks of the same family produces a shock of that family plus a rarefaction wave of the opposite family. Using Theorem 1 we can therefore formulate this condition by requiring that the quantities in (14) and (15) be positive.

Acknowledgement. This research was supported in part by N. S. F. Contract No. 01048. 


\section{References}

1. Cesari, L., Sulle funzioni a variazione limita. Ann. Scuola Norm. Pisa (2), 5, 299-313 (1936).

2. Dieudonné, J., Foundations of modern analysis. Academic Press 1960.

3. De GroRgi, E., Nuovi teoremi relativi alle misure $(n-1)$-dimensionale in uno spazio ad $\mathbf{r}$ dimensioni. Richerche Mat. 36, 95-113 (1955).

4. Gurmm, J., Solutions in the large for non-linear hyperbolic systems of equations. Comm. Pure Appl. Math. 18, 695-717 (1965).

5. Glimm, J., \& P. D. LAx, Decay of solutions of systems of hyperbolic conservation laws. Bull. A. M. S. 73, 105 (1967).

6. Fleming, W. H., Functions with generalized gradient and generalized surfaces. Ann. Mat. Pura Appl. ser. 4, 44, 93-104 (1957).

7. Johnson, J. L., \& J. A. Smoller, Global solutions of certain hyperbolic systems of quasilinear equations. J. Math. Mech. 17, 561- 576 (1967).

8. Johnson, J. L., \& J. A. SMOLleR, Global solutions of hyperbolic systems of conservation laws in two dependent variables. To appear in Bull. A. M. S.

9. LAx, P. D., Hyperbolic systems of conservation laws, II. Comm. Pure Appl. Math. 10, 537-566 (1957).

10. LAX, P. D., Development of singularities of solutions of nonlinear hyperbolic partial differential equations. J. Math. Ph. 5, 611-613 (1964).

11. Zhang ToNG \& Guo YU-FA, A class of initial-value problems for systems of aerodynamic equations. Acta Math. Sinica 15, 386- 396 (1965). English translation in Chinese Math. 7, 90- 101 (1965).

The University of Michigan Ann Arbor 